\title{
La participación de las familias en el análisis y la transformación de su realidad mediante un debate familiar sobre valores y convivencia*
}

\author{
The Participation of Families in the Analysis and Transformation of their \\ Reality through a Family Debate about Values and Living Together
}

Recibido: diciembre 23 de 2011 | Revisado: agosto 19 de 2014 | Aceptado: agosto 19 de 2014

\author{
Paz Viguer Seguí ** \\ NEUS SOLÉ PÉREZ *** \\ Universitat de València, España
}

doi:10.11144/Javeriana.upsy13-5.lpfa

Para citar este artículo: Viguer, P. \& Solé, N. (2015) La participación de las familias en el análisis y la transformación de su realidad mediante un debate familiar sobre valores y convivencia. Universitas Psychologica, 14(1), 355-366. http://dx.doi. org/10.11144/Javeriana.upsy13-5.lpfa

Agradecemos la participación activa de las casi 500 familias que con su reflexión y diálogo intrafamiliar en torno a los valores y la convivència han permitido la realización de este estudio.

** Profesora titular de la Universidad de Valencia. Correo electrónico: paz.viguer@uv.es

**** Licenciada en Psicología. Investigadora. Correo electrónico: neusope@alumni.uv.es

\section{RESUMEN}

Este estudio aborda la socialización en valores y convivencia a partir de una investigación acción que parte de un enfoque participativo y dialéctico y que utiliza el Debate familiar, una herramienta innovadora cuyas finalidades son favorecer la reflexión y el diálogo intrafamiliar e implicar a las familias en el análisis y transformación de su realidad. Esta estrategia estimula el debate e invita a pensar sobre los valores presentes en la familia, recoge información de los diferentes miembros de la unidad familiar y a su vez, sensibiliza sobre los temas tratados. Un total de 492 familias, con al menos un hijo o hija entre 7 y 16 años, participaron en este estudio.

Los resultados ponen de relieve la potencialidad del instrumento como recurso facilitador de la identificación de problemáticas reales o potenciales y elementos y dinámicas positivas; y su utilidad para implicar a la familia y a la comunidad en su análisis y la formulación de líneas de acción para su transformación. Asimismo, los resultados permiten la formulación de líneas de acción comunitarias que favorecen la construcción de una convivencia positiva.

Palabras clave

convivencia; debate familiar; metodología dialéctica; participación comunitaria; socialización

\section{A B S T R A C T}

This study was aimed at socializing values and coexistence from an action research from a participatory and dialectical approach and uses the familiar debate, an innovative tool whose aims are to promote reflection and domestic dialogue and involve families in the analysis and transformation of reality. This strategy encourages debate and invites us to think about the values present in the family, collects information from different members of the family unit and in turn, raises awareness of the issues. A total of 492 families with at least one child between 7 and 16 years, participated in this study. The results highlight the potential of the instrument as a facilitator resource positive identification of actual or potential problems and elements and dynamics; and its usefulness to involve the family and the community in the analysis and formulation of action lines for processing. Moreover, the results allow the formulation of Community action lines which foster the building of a positive coexistence.

Keywords

Coexistence; family discussion; dialectic methodology; comunity participation; socialization 


\section{Introducción}

La promoción de la convivencia pacífica se ha convertido en un reto de primer orden cuya finalidad es mejorar las relaciones entre los individuos. El diálogo, la negociación, la cooperación y el respeto constituyen metas educativas y estrategias eficaces tanto para la prevención de conflictos como para la solución no violenta de los mismos. Dado que conflicto y convivencia son dos realidades sociales inherentes a toda forma de vida en sociedad, se considera imprescindible capacitar a los sujetos para resolver los conflictos de manera pacífica y promover relaciones saludables; y se apuesta por un modelo de política global en el que todos los contextos se ven involucrados en la mejora de la convivencia y la prevención de la violencia (Viguer \& Avià, 2009).

En este marco y a finales de 2004, la Secretaria de Juventud de la Generalitat de Catalunya impulsó una línea de trabajo en el ámbito de la prevención comunitaria centrada en las relaciones abusivas. Una de las actuaciones que se está llevando a cabo desde 2006 es la implementación de un proyecto de prevención de las violencias que afectan a los y las jóvenes (Viguer, Avià, \& Rossell, 2013). Este proyecto se puso en marcha como experiencia piloto en tres municipios con el objetivo de promover modelos de relación igualitarios, facilitar el diálogo y la resolución no violenta de conflictos y elaborar un protocolo que facilite la detección, coordinación e intervención en situaciones de riesgo y/o violencia.

Una acción concreta llevada a cabo fue el debate familiar sobre valores y convivencia en Castellar del Vallés (DFVCC). El Debate familiar es un instrumento que se ha utilizado en estudios participativos sobre temas como el estado actual del municipio y cómo se desea en un futuro (Ajuntament de St. Feliu de Llobregat, 2002), las actividades extraescolares (Fundació Jaume Bofill, 2005), los valores y la convivencia (Consell Comarcal del Bages \& Universitat de València, 2008) o la coeducación (Ajuntament de Calella, 2009) y cuya eficacia ha sido mostrada en en los trabajos de Viguer y Solé (2011, 2012) y Viguer, Rodrigo y Solé (2013). Los propósitos fundamentales del Debate familiar son favorecer la reflexión y el diálogo intrafamiliar en torno a una temática concreta, recoger información que permita identificar problemáticas reales o potenciales y elementos y dinámicas positivas, y dinamizar a la comunidad educativa en el análisis y la transformación social de su propio entorno.

La dinamización social constituye un proceso clave en la prevención comunitaria ya que implica tanto la participación como el sentimiento de corresponsabilidad en la respuesta a los problemas por parte de la población y de los diferentes agentes implicados. La evidencia empírica ha demostrado que la implicación de la comunidad y la mejora de sus capacidades contribuyen a una mayor calidad de la prevención y conducen a resultados positivos en las intervenciones (Chinman et al., 2005). En este sentido, el Debate familiar promueve la participación de una de las instituciones más relevantes de la comunidad: la familia.

La familia es un espacio educativo privilegiado en el que se transmiten modelos de relación y convivencia de padres a hijos/as y en el que el niño desarrolla procesos cognitivos, afectivos y conductuales a través de la socialización. La socialización es el proceso mediante el cual el sujeto interactúa con una red de agentes y contextos a través de los cuales adquiere los valores, normas, creencias y formas de conducta que son esenciales para su participación eficaz en la sociedad. Aunque los agentes que intervienen en la socialización son múltiples, la familia suele ser el más importante, sobre todo en los primeros años de vida, ya que los niños son especialmente moldeables y sus destrezas sociales, valores y competencias comienzan a formarse a partir, fundamentalmente, de la observación y de la imitación de los progenitores (Pichardo, Justicia, \& Fernández, 2009).

A medida que los niños crecen pasan más tiempo en contextos extra familiares y parece ser que la influencia del grupo de iguales aumenta. Sin embargo, a pesar de la importancia que tiene el vínculo con los iguales y de que les hace más caso en algunos temas (como el ocio o el vestuario), los jóvenes siguen considerando la opinión de los padres en cuestiones relevantes como el dinero, la moral, la elección de estudios, etc. (Navas, Torregrosa, \& Mula, 2004). 
Ahora bien, la socialización no es una vía de sentido único en el que los hijos tienen un papel pasivo, sino un proceso bidireccional en el que cada miembro de la familia puede influir en el otro, en su conducta, actitudes, sentimientos y valores (Musitu, Jiménez \& Povedano, 2009). Sin embargo, la mayor parte de las investigaciones se han dirigido a estudiar los correlatos existentes entre las estrategias educativas de los progenitores y el comportamiento infantil. Fruto de estos trabajos, se han establecido cuatro estilos educativos en base a las dimensiones afecto y control-exigencias (autoritario, democrático, indulgente y negligente) y se ha determinado que, en el contexto español, una educación orientada hacia el afecto y la comunicación (diálogo, explicación y razonamiento) favorece el ajuste psicosocial de los hijos/as (Musitu \& García, 2004) y es más eficaz que una educación orientada hacia la coerción (Musitu, Estévez, Jiménez, \& Herrero, 2007).

En cuanto a la prevalencia de cada uno de los estilos en las familias españolas, un estudio realizado por Pichardo (2000) reveló que el estilo democrático es el más utilizado (53\%), seguido del permisivo (32\%), el autoritario (9\%) y el negligente (4\%).

Por otra parte, como afirman Darling y Steinberg (1993), es importante tener en cuenta las metas y valores de los padres, las prácticas educativas que emplean y las actitudes educativas que manifiestan a sus hijos/as. En consecuencia, en el proceso de socialización cobran especial relevancia el componente cognitivo (las creencias y valores de los progenitores sobre el desarrollo y la educación) y el conductual (el comportamiento de los progenitores en situaciones concretas). Cabe considerar que de acuerdo con Rodrigo y Palacios (1998), el binomio cognición-acción no implica una correspondencia perfecta entre ambos, por tanto para su conocimiento sería recomendable utilizar varios métodos de evaluación convergentes, directos e indirectos. Esta afirmación se encuentra respaldada por un estudio relacionado con los estilos educativos de los progenitores (qué valores trataban de instaurar, qué normas tenían en casa, qué hacían para que sus hijos cumplieran las normas, etc.), que evidenció que los progenitores presentaban creencias bastante adecuadas en relación a cómo debe ser la educación de sus hijos, pero que encontraban problemas a la hora de llevarlas a la práctica (Hernández, Gómez, Martín, \& González, 2008). En esta línea, el estudio de Viguer y Solé (2011) evidenció que la mayoría de los progenitores presentaban valores y creencias relacionados con la crianza propios de un estilo democrático, al analizarlos de manera directa mediante un cuestionario. Sin embargo, 63,1\% de los progenitores presentaban un estilo autoritario al analizar de manera indirecta su comportamiento ante un caso práctico.

Si atendemos a la investigación relacionada con los valores familiares, los estudios realizados en contextos españoles han evidenciado que la mayoría de familias trata de inculcar a sus hijos/ as valores que posibiliten una convivencia en paz (tolerancia, respeto a los demás y responsabilidad) y valores relacionados con el esfuerzo y el trabajo (Meil, 2006), mientras que el éxito, entendido como la importancia de conseguir dinero, fama e importancia social, genera controversia (Viguer \& Solé, 2011). Por otra parte, se observa que $99 \%$ de los españoles consideran que la familia es uno de los valores más importantes, por encima del trabajo, los amigos, el tiempo libre, la religión y la política (Elzo \& Orizo, 2000).

En cuanto a la juventud española, se observa que los jóvenes mantienen como valores prioritarios la familia y los amigos, seguidos por el trabajo, ganar dinero, el tiempo libre, llevar una vida moral y digna, tener una vida sexual satisfactoria, los estudios para tener una buena formación y competencia profesional y en último lugar, la política y la religión (González Blasco, et al., 2006). A pesar de que los progenitores en general perciben una pérdida de autoridad (Meil, 2006), desde la perspectiva de los hijos, parece ser que la familia sigue siendo la institución más importante.

Por otra parte, en cuanto a aspectos como la aceptación de la diversidad, parece ser que la influencia de los padres es la más importante, como muestra el estudio de Navas et al. (2004) en el que las actitudes hacia la integración parecen depender en mayor medida de las actitudes de los padres y de las conversaciones mantenidas con ellos sobre el 
tema, que del conocimiento mutuo de compañeros con necesidades educativas especiales o de las actitudes de los iguales.

Sin duda, la familia es una de las instituciones más influyentes en el aprendizaje de valores y modelos de convivencia. En consecuencia, el análisis de estos temas dentro del núcleo familiar cobra una gran relevancia. La investigación participativa constituye un modelo de dinamización territorial que crea el marco adecuado para que la comunidad sea partícipe de un proceso de reflexión y construcción de conocimiento praxeológico, socialmente útil, además de constituir un instrumento para la toma de conciencia colectiva y una acción de autopromoción, mejora de la calidad de vida local (Basagoiti \& Bru, 2000) y cambio a partir del diseño e implementación de intervenciones y propuestas socioeducativas. Las metodologías participativas se basan en la perspectiva dialéctica como eje de la investigación y pretenden transformar las situaciones no solo con la práctica, sino con la reflexión sobre esa práctica. Por tanto, si partimos de las técnicas dialécticas de intervención debemos considerar al objeto a investigar como sujeto protagonista de la investigación (Alberich, 2000).

Desde esta perspectiva, consideramos de gran importancia realizar estudios contextualizados que promuevan la implicación de los individuos en el análisis de su realidad, en cuanto a la transmisión de valores y la convivencia, y en la construcción de una convivencia positiva (Viguer \& Avià, 2009). Por ello, este trabajo presenta parte de los resultados de una investigación realizada a partir del DFVCC, una estrategia que permitió recoger información mediante la participación activa de las familias en el análisis de su realidad. Sus objetivos fueron promover la reflexión y el diálogo intrafamiliar en torno a los valores y la convivencia; recoger la visión de la familia sobre valores y convivencia en los diferentes contextos de socialización (familia, escuela y comunidad), implicar a las mismas en el proceso de análisis y de transformación de su realidad y, a su vez, sensibilizar y dinamizar a la comunidad educativa sobre el tema, devolviendo posteriormente los resultados del diagnóstico de la población para invitar a las familias a entrar en debate y formular propuestas. El objetivo principal de este artículo es presentar los resultados relativos a la socialización familiar. De forma más específica, pretendemos explorar las cuestiones del debate relativas a: estilos y valores educativos, valores familiares, obediencia a la autoridad, establecimiento de normas y aceptación de la diversidad.

\section{Método}

\section{Participantes}

En esta investigación participaron 492 familias con al menos un hijo o hija entre los siete y dieciséis años, escolarizados en Castellar del Vallès. Este municipio tiene entre 20000 y 25000 habitantes, un perfil industrial y de prestación de servicios y se localiza en la provincia de Barcelona (España). En la fecha en la que se pasó el cuestionario había 1876 familias en el municipio que tenían un hijo/a en esta franja de edad, por lo tanto se recogió información correspondiente al 26,3\% de las familias.

Respecto al tipo de familias, $93.67 \%$ son familias en las que ambos progenitores conviven en la unidad familiar y en $6.33 \%$ restante solo uno. El número de hijos/as oscila entre 1 y $5(\mathrm{M}=1$ '8). Los progenitores tienen una media de edad de 41 años $(D T=5.63)$ y un rango de 28 a 55 años. Respecto a su nivel educativo: $13,75 \%$ tiene estudios primarios, $14,1 \%$ secundarios, $15,9 \%$ bachillerato, $24,95 \% \mathrm{FPy}$ $31,3 \%$ estudios superiores. Respecto al número de niños/as participantes, se contabilizan 841 sujetos, siendo $56 \%$ niñas y $44 \%$ niños.

\section{Instrumento}

Debate Familiar sobre valores y convivencia en Castellar del Vallès (DFVCC): instrumento elaborado ad hoc para recabar información sobre los valores y formas de convivencia presentes en la familia, la escuela y la comunidad. El debate familiar rompe con los clásicos instrumentos de recogida de datos y se plantea como una nueva forma de recoger información que a su vez, permite sensibilizar a las familias respecto a los temas tratados. Este instrumento contiene una introducción en la que 


\section{TABLA 1}

Dimensiones (modalidades de respuesta) e items del DF presentados en este trabajo

- Estilos educativos (a): A partir de unas viñetas en las que aparece un conflicto cotidiano entre padre-hijo, explicar cómo se resolvería en la propia familia.

- Valores educativos (b): Señalar con una cruz si están bastante, poco o nada de acuerdo con diferentes afirmaciones propuestas relacionadas con la crianza.

- Valores familiares (a):

- Valorar en una escala de 1 a 10 la importancia que otorgan a diez valores (honestidad, paz, esfuerzo, éxito, tolerancia, diálogo, obediencia, ayudar a los/las demás, respeto y libertad).

- Ordenar ocho instituciones presentadas según la importancia que se les atribuye, siendo 1 la que consideran más importante y 8 la que consideran menos importante (gobierno, escuela, familia, médicos, iglesia, televisión-radio-periódicos, asociaciones-clubs deportivos y ejército).

- Obediencia a la autoridad (c): Indicar con una cruz si hacen mucho, bastante o poco caso al padre, a la madre, a los hermanos/as mayores y a los abuelos/as.

- Establecimiento de normas (b): Indicar con una cruz si las normas en casa las pone el padre, la madre, ambos progenitores o si se negocian entre todos.

- Aceptación de la diversidad (d): Señalar con una cruz si tienen amigos/as de otro país, de otra religión, con una discapacidad y/o con ideas muy opuestas a las suyas.

Nota: modalidades de respuesta: (a) todos juntos; (b) solo el padre y la madre; (c) cada hijo/a individualmente y (d) cada miembro de la familia individualmente.

Fuente: elaboración propia

se presenta y explica el instrumento a las familias, se recuerda que es anónimo y se solicita su sinceridad. El DFVCC consta de un cuadro que recoge los datos sociodemográficos básicos (el número de miembros que conviven en la unidad familiar, su edad, el género de los hijos y el nivel de estudios de los progenitores) y está formado por veinte ítems agrupados en tres bloques temáticos (socialización en la familia, en la escuela y en la comunidad). En la Tabla 1 se detallan las áreas temáticas, las dimensiones y los ítems que aborda este trabajo.

Según el contenido de los ítems hay diferentes modalidades de respuesta: (a) contesta toda la familia consensuando una respuesta, (b) contestan solo los progenitores consensuando una respuesta, (c) contestan solo los hijos/as por separado, (d) cada miembro de la familia contesta por separado. El formato de respuesta es combinado: algunos ítems contienen preguntas abiertas y otros preguntas cerradas (de simple selección, de elección múltiple, de jerarquización, escala tipo Likert...).

Esta modalidad de presentación de las cuestiones, junto con un diseño atractivo a nivel visual, facilita que los participantes mantengan la atención y la motivación hacia la tarea. Además, el instrumento contiene cuadros con mensajes que transmiten información relevante sobre aspectos de la convivencia. Se trata de elementos educa- tivos y sensibilizadores que inducen al debate y la reflexión, al tiempo que inciden sobre la importancia de la educación familiar y de la participación comunitaria.

\section{Procedimiento}

Una vez elaborado el instrumento se realizó una prueba piloto mediante la administración del cuestionario a ocho familias, elegidas en base a una serie de criterios para garantizar la máxima heterogeneidad posible (condición social, procedencia, nivel formativo, modelo familiar, número de miembros y edad de los hijos/as). Tras la realización del debate se realizó una entrevista con cada familia para recoger su valoración del cuestionario así como las dificultades observadas. A partir de la información recabada se revisó el cuestionario incorporando las propuestas y sugerencias. Seguidamente se distribuyó el cuestionario final a través de los centros educativos. El profesorado de los centros se encargó de repartir los debates al alumnado, un dinamizador externo pasó por todas las aulas y explicó en qué consistía el proyecto y cómo debía cumplimentarse el instrumento y se dio un plazo de quince días para que lo rellenaran con su familia. Una vez recogidos todos los cuestionarios se remitieron a la universidad en la que se realizó la codificación y el 
análisis estadístico de datos, utilizando el paquete estadístico SPSS.15. Para el análisis de los datos, efectuado a un nivel de confianza del $95 \%$ y con un margen de error del $5 \%$, se han utilizado estadísticos descriptivos y la prueba chi cuadrado.

\section{Resultados}

Los resultados correspondientes a los estilos educativos muestran que el porcentaje de familias que presenta un estilo autoritario en un caso práctico en el que deben resolver un conflicto familiar $(60,8 \%)$ es superior a la proporción de familias que presenta un estilo democrático (29,4\%), permisivo $(6,6 \%)$ o negligente (3,2\%). En cambio, si nos centramos en los valores relacionados con las prácticas educativas (Tabla 1), observamos que $93,8 \%$ de los progenitores se muestra bastante de acuerdo con que los hijos tienen que ser criados en brazos, con mucho contacto físico y afecto, y si puede ser con lactancia natural y 97,2\% con que es importante jugar, hablar y hacer cosas junto con los hijos.

Igualmente se observa que $61,6 \%$ de los progenitores no está nada de acuerdo con que 'con los niños no se debe razonar: primero deben aprender a obedecer' y 26,3\% está poco de acuerdo. Solamente $12,1 \%$ se muestra de acuerdo con esta afirmación. Por tanto, la mayoría de los progenitores consideran que se debe dialogar y razonar con los hijos antes de imponerles su autoridad.

En relación con si una bofetada a tiempo puede ser positiva, $+33,4 \%$ de los progenitores se muestra bastante de acuerdo. En la tendencia contraria encontramos $42,8 \%$ que está poco de acuerdo y 23,8\% que no lo está nada. También podemos observar que 39\% de los progenitores está bastante de acuerdo con que 'vivimos en un mundo competitivo y lo más importante es ayudar a los hijos/ as a tener éxito', frente a 42,5\% que está poco de acuerdo y $18,5 \%$ que no lo está nada. Por otra parte, la mayoría de los progenitores (84\%) está bastante de acuerdo con que 'muchos niños de hoy en día están mal educados' y 39,4\% de los progenitores está bastante de acuerdo con que 'hacer demasiado caso a los niños/as los malcría', frente a 37,6\% que está poco de acuerdo y $23 \%$ que no lo está nada. En cuanto al papel de la escuela, la mayor parte de los progenitores se muestra en desacuerdo con que esta institución es la que básicamente, debe educar a los hijos $(94,8 \%$ frente a 5,2\% que está bastante de acuerdo) y con que la escuela no debe educar, si no enseñar materias $(72,4 \%$ frente a $27,6 \%$ que está bastante de acuerdo).

Tal como podemos observar en la Tabla 2, los valores ordenados de mayor a menor en base a la importancia que le atribuyen las familias son: paz,

TABLA 1

Valores educativos expresados en frecuencias absolutas

\begin{tabular}{lccc}
\hline & BD & PD & ND \\
\hline Los hijos tienen que ser criados en brazos, mucho contacto físico y afecto, y si puede & 93.8 & 5.1 & 1.1 \\
ser con lactancia natural & 39.4 & 37.6 & 23 \\
Hacer demasiado caso a los niños/as los malcría & 33.4 & 42.8 & 23.8 \\
A veces una bofetada a tiempo es positiva & 12.1 & 26.3 & 61.6 \\
Con los niños no se debe razonar: primero deben aprender aobedecer & 84 & 14.5 & 1.5 \\
Muchos niños de hoy en día están mal educados & 39 & 42.5 & 18.5 \\
Vivimos en un mundo competitivo y lo más importante es ayudar a los hijos a tener & 97.2 & 17 & 1.1 \\
éxito & 5.2 & 40.9 & 53.9 \\
Es importante jugar, hablar y hacer cosas junto con los hijos & 27.6 & 40.7 & 31.7 \\
La escuela es la que, básicamente, debe educar a los hijos & & & \\
La escuela no debe educar, sino enseñar: mates, lengua, ciencias, orden, técnicas de & estudio & &
\end{tabular}

BD: bastante de acuerdo, PD: poco de acuerdo, ND: nada de acuerdo

Fuente: elaboración propia 
TABLA 2

Media y desviación de la importancia otorgada a diez valores

\begin{tabular}{lcc}
\hline \multicolumn{1}{c}{ Valores } & M & (DT) \\
\hline Honestidad (no podemos engañar, mentir, robar, decir falsedades de otros/as...) & 9.33 & $(1.60)$ \\
Paz (queremos un mundo sin violencia) & 9.65 & $(1.43)$ \\
Esfuerzo (en la vida tenemos que esforzarnos para conseguir lo que queremos) & 9.29 & $(1.51)$ \\
Éxito (es importante conseguir dinero, fama e importancia social) & 5.09 & $(2.58)$ \\
Tolerancia (tenemos que ser capaces de convivir con personas diferentes a nosotros - por país, ideas, & 8.74 & $(1.87)$ \\
religión, orientación sexual, estado de salud...-) & 9.24 & $(1.50)$ \\
Diálogo (solo hablando y negociando se arreglan los conflictos) & 7.12 & $(2.54)$ \\
Obediencia (es importante obedecer las órdenes que se nos dan) & 8.64 & $(1.83)$ \\
Ayudar a los demás (debemos ayudar tanto a los que están cerca como a quienes no conocemos y nos \\
necesitan)
\end{tabular}

Fuente: elaboración propia

honestidad, esfuerzo, diálogo, respeto, libertad, tolerancia, ayudar a los demás, obediencia y éxito.

Los datos obtenidos muestran que la familia es la institución a la que las familias dan más importancia, seguida por la escuela y por los médicos (Tabla 3). En la cuarta posición encontramos el gobierno (Estado, generalidad y ayuntamiento), en la quinta los clubs deportivos y asociaciones y a continuación, la televisión y la radio. En penúltima posición aparece la iglesia y en última, el ejército.

Como se puede observar en la Tabla 4, la proporción de hijos/as que afirma hacer mucho caso a los abuelos/as es superior a la de los que afirman hacer mucho caso a los padres y a las madres. Se observa también que el número de hijos/as que afirman hacer poco caso a los abuelos es superior al que los que afirman hacer poco caso a las madres y/o a los padres y que a los hermanos mayores es a quien menos caso hacen.

Verificamos diferencias estadísticamente significativas en la variable obediencia a la autoridad: los niños/as obedecen más a los padres que a las madres $\left(\chi^{2}=322.072, \mathrm{gl}=4, \mathrm{p}=0.000\right)$, que a sus abuelos/as $\left(\chi^{2}=67.320, \mathrm{gl}=4, \mathrm{p}=0.000\right)$ y que a sus hermanos/as $\left(\chi^{2}=66.842, \mathrm{gl}=4, \mathrm{p}=0.000\right)$. Asimismo, observamos que las niñas obedecen más a las madres que los niños $\left(\chi^{2}=3.788, \mathrm{gl}=2, \mathrm{p}=\right.$ 0.150), sin embargo no encontramos diferencias de género significativas en la obediencia a los padres $\left(\chi^{2}=0.586, \mathrm{gl}=2, \mathrm{p}=0.746\right)$, a los abuelos/as $\left(\chi^{2}=0.282, \mathrm{gl}=2, \mathrm{p}=0.868\right)$ o a los hermanos/ as $\left(\chi^{2}=0.860, g l=2, p=0.650\right)$.

TABLA 3

Media y desviación típica de la importancia otorgada a ocho instituciones

Instituciones

$\mathrm{M}(\mathrm{DT})$

Gobierno (Estado, Generalitat y Ayuntamiento)

$4.37(1.39)$

Escuela

$2.53(0.93)$

Familia

$1.15(0.75)$

Médicos

$2.95(0.93)$

Iglesia

$6.63(1.40)$

Televisión, radio, periódicos

$5.62(1.19)$

Asociaciones y clubs deportivos

$5.42(1.31)$

Ejército

$7.29(1.16)$

Fuente: elaboración propia 
TABLA 4

Grado de obediencia a los familiares expresado en frecuencias absolutas

\begin{tabular}{lccc} 
& Mucho & Bastante & Poco \\
\hline Al padre & 41 & 50.6 & 8.4 \\
A la madre & 40 & 48.8 & 11.2 \\
A los hermanos/as mayores & 10.7 & 29.3 & 60 \\
A los abuelos/as & 43.9 & 42.7 & 13.4 \\
\hline
\end{tabular}

Fuente: elaboración propia

TABLA 5

Frecuencias absolutas de los miembros de la familia con amistades atendiendo a la diversidad

\begin{tabular}{ccccc} 
& De otro país & De otra religión & Con una discapacidad & Con ideas muy opuestas \\
\hline Niños/as & 79.8 & 52.1 & 48.6 & 20.7 \\
Madre & 68.4 & 55.3 & 59.5 & 6.3 \\
Padre & 73.5 & 55 & 55.6 & 63.4 \\
\hline
\end{tabular}

Fuente: elaboración propia

Respecto a cómo se ponen las normas del hogar, $1,3 \%$ de los progenitores afirma que las pone el padre, $12,2 \%$ indica que las pone la madre, $58,8 \%$ señala que las ponen ambos progenitores y $27,7 \%$ afirma que las negocian entre todos/as.

En cuanto a la proporción de amistades con características específicas que progenitores e hijos/ as tienen (Tabla 5), los resultados revelan que la proporción de niños/as con amigos/as de otro país es superior a la de padres y madres que los tienen. Sin embargo la proporción de niños/as con amigos/as con una discapacidad o con ideas muy opuestas a las suyas es inferior a la de padres y madres que afirman tenerlos. Por último, respecto a las amistades de otra religión que tiene cada miembro de la familia vemos que los resultados son bastante similares: $52,1 \%$ de los hijos/as, 55,3\% de los padres y 55\% de las madres afirman tenerlas. Estos datos arrojan diferencias significativas: los hijos e hijas tienen más amistades de otro país que sus progenitores $\left(\chi^{2}=82.714, \mathrm{gl}=1, \mathrm{p}\right.$ $=0.000)$ y menos amistades con ideas muy opuestas a las propias $\left(\chi^{2}=38.400, \mathrm{gl}=1, \mathrm{p}=0.000\right)$, con una discapacidad $\left(\chi^{2}=187.971, \mathrm{gl}=1, \mathrm{p}=0.000\right)$ y de otra religión $\left(\chi^{2}=126.863, \mathrm{gl}=1, \mathrm{p}=0.000\right)$.

\section{Discusión}

De los resultados obtenidos respecto a la socialización familiar pueden destacarse diversos aspectos. En primer lugar, los datos apuntan a que el estilo autoritario es el más utilizado en las familias participantes y no apoyan los obtenidos por Pichardo (2000) sobre la prevalencia del democrático. Sin embargo, las medidas relativas a los valores educativos muestran que, en general, los progenitores consideran importante el afecto, el diálogo, el razonamiento y el compartir actividades con los hijos, valores próximos al estilo democrático, a pesar de que una proporción destacable ve el uso de una bofetada como algo positivo.

A su vez, vemos que los valores educativos no se corresponden con la práctica dado que en la resolución de un conflicto, la mayor parte de los progenitores utiliza un estilo autoritario, es decir que impone sus reglas sin dar lugar al diálogo. Estos resultados son similares a los obtenidos por Álvarez González (2006) en los que la gran mayoría de los entrevistados (95\%) consideran el diálogo como la mejor actitud de los progenitores ante situaciones comprometidas y $60,1 \%$ opina que un azote a tiempo evita problemas mayores. Teniendo en cuenta estos datos y de acuerdo con Jares (2006) consideramos fundamental promover el aprendizaje de estrategias eficaces de resolución de conflictos y de técnicas de disciplina positiva, para evitar que se recurra a la llamada violencia de baja intensidad y se justifique su uso. 
En segundo lugar, a la vista de los resultados se desprende la importancia de distinguir entre las creencias educativas y las prácticas concretas dado que en general, se constata la presencia de creencias bastante adecuadas en relación con la educación de los hijos pero no sucede lo mismo con la praxis, tal y como ocurre en el estudio de Hernández et al. (2008). Resaltamos la utilidad del instrumento utilizado para realizar evaluaciones tanto directas como indirectas y así obtener datos sobre cogniciones y sobre conductas. Y aunque idealmente cabría esperar una mayor correspondencia entre el binomio cognición-acción, la realidad reitera que no se produce una correspondencia perfecta. Por tanto, consideramos que se debería seguir trabajando para analizar las relaciones entre creencias y conducta y desvelar los procesos que explican dichas relaciones. También es importante que los progenitores reflexionen y expliciten sus propias creencias, para que sean conscientes de sus concepciones y se encuentren en disposición de cambiarlas, si fuera necesario (Rodrigo \& Palacios, 1998).

Pensamos que sería conveniente promover la participación de los progenitores en programas educativos a nivel local, ya que el entrenamiento estructurado y sistemático en pautas de comportamiento es un recurso útil para proporcionar conocimientos y destrezas para afrontar adecuadamente situaciones concretas en relación con los hijos. Este tipo de intervenciones pueden aportar apoyo y recursos, además de promover en ellos sentimientos de competencia y seguridad y una mayor coherencia entre sus propias creencias y conductas.

En tercer lugar, es de destacar que la mayoría de progenitores participantes consideran que escuela y familia comparten la responsabilidad de educar a los hijos/as, aunque una pequeña parte atribuye dicha responsabilidad principalmente a la escuela y otros le eximen de ella. Además, se observa que gran parte de los progenitores opinan que muchos niños de hoy en día están mal educados. En este punto cobra relevancia la necesidad de colaboración entre familia y escuela para asegurar una educación de calidad. Así pues, debería incentivarse la participación de las familias en los centros educativos para abordar conjuntamente aspectos relativos a la educación, dado que como señala Ribes (2002), la colaboración entre ambas instituciones aporta beneficios que redundan en un mejor desarrollo infantil. Entre ellos podemos destacar el establecimiento de criterios educativos comunes, la posibilidad de ofrecer modelos de relación e intervención, la divulgación de la función educativa de la escuela para aumentar la comprensión, la aceptación y la valoración de su labor y para evitar confusiones de roles y competencias; y el enriquecimiento de la escuela con las aportaciones de las familias como recurso humano de apoyo y como posibilidad de reflexionar de manera conjunta y obtener una opinión complementaria.

Por lo anterior proponemos que se fomenten las relaciones entre familia y escuela con iniciativas atractivas y motivadoras que ofrezcan posibilidades que se ajusten a la disponibilidad de ambas partes y así favorezcan su participación. Algunas acciones concretas para incentivar la co-responsabilidad podrían consistir en ofrecer tiempo y espacios para cultivar la convivencia a través de contactos formales e informales, fomentar la participación conjunta en la planificación de las acciones educativas favoreciendo iniciativas como las comunidades de aprendizaje (Flecha \& Puigvert, 2002), las full service community schools (Dryfoss \& Macguire, 2002), o las redes locales de debate educativo (Comellas, 2006).

En cuarto lugar por lo que respecta a los valores familiares, observamos que las familias otorgan mucha importancia al esfuerzo dirigido hacia la consecución de una meta y a los valores de convivencia positivos, sin embargo el valor éxito genera controversia. Estos resultados siguen la línea de los obtenidos por Meil (2006) y Viguer y Solé (2011, 2012) y Viguer, Rodrigo y Solé (2013). No obstante sería interesante evaluar si esos valores realmente se practican, mediante la utilización de métodos de evaluación indirectos que permitan obtener información sobre la praxis de los valores en la familia. Además, se observa que en consonancia con Elzo y Orizo (2000) y González Blasco et al. (2006), la familia aparece como un valor prioritario, por encima de la escuela, los médicos y el gobierno, entre otros. Por lo que podemos afirmar que a pesar de que en la actualidad se habla de crisis de la familia 
(Ortega \& Minguez, 2001), esta sigue siendo la institución social principal para sus propios miembros.

También observamos que los hijos/as tienen más amistades de otro país que sus progenitores y menos amigos/as con una discapacidad, de otra religión o con opiniones muy opuestas a las propias. Estos resultados parecen transmitir la realidad actual de las sociedades occidentales en las que sucesivos flujos migratorios masivos han provocado la formación de grupos étnicos y culturales diferenciales y estables, pasando a convertirse en sociedades étnicamente plurales. La multiculturalidad existente empieza su camino hacia la interculturalidad a través de la integración en las aulas y la consiguiente aparición de vínculos de amistad intercultural.

Si consideramos el valor que se otorga a la familia, que cada uno de sus miembros puede influir en los valores, actitudes y conducta del resto de miembros y que la integración podría predecirse por las actitudes de los progenitores y de las conversaciones que con ellos se mantienen, debería seguirse trabajando a este nivel para favorecer la aceptación y el respeto por la diversidad desde una perspectiva intergeneracional. Así, los progenitores podrían favorecer en mayor medida la tolerancia hacia la discapacidad y la diferencia ideológica, bien sea política o religiosa, y los hijos podrían favorecer el conocimiento y la aceptación de la diversidad étnica y cultural.

Proponemos que se desarrollen acciones conjuntas no solo a nivel familiar sino a nivel comunitario, como la organización y coordinación de actividades e intervenciones con organismos oficiales y otras entidades que ofrezcan información sobre las oportunidades que ofrece la diversidad y que favorezcan el contacto, el aprendizaje y la cooperación, a partir del respeto, con personas diferentes en relación al género, la edad, la raza, las capacidades, la religión, etc.

En quinto y último lugar, se destaca que en la mayor parte de las familias las normas son impuestas por los progenitores sin hacer partícipes a los hijos en la elaboración de las mismas. Esta práctica se asienta en la tradición autoritaria de imponer la obediencia de los hijos a unas normas preestablecidas por los progenitores. Evidentemente, es imprescindible marcar unas pautas y unos límites claros a los hijos ya que toda convivencia exige un marco de normas básicas que la regulen. La cuestión no es la necesidad de disciplina sino el modo en que se ejerce. Por ello las normas deberían ser debatidas y consensuadas, ya que al hacer partícipes a todos los implicados en la reflexión, razonamiento y consenso de las normas, al sentirlas como propias, se favorece tanto su aceptación como la de las consecuencias que se derivan de su incumplimiento.

Los procedimientos educativos de la democracia participativa están basados en estas premisas y han demostrado su eficacia en intervenciones concretas (Díaz Aguado, 2003). El debate de las normas podría contribuir a disminuir la pérdida de autoridad señalada por Meil (2006) y a modificar la opinión de que muchos niños de hoy en día están mal educados. Así, se observa nuevamente la necesidad de trabajar con los progenitores las bases de estas estrategias de democracia participativa para poder utilizarlas en el contexto familiar.

En este estudio, el DFVCC se ha utilizado con la finalidad de facilitar el diálogo intrafamiliar y de recoger información relativa a las familias para implicarlas en el análisis de su realidad. Debemos señalar que esta acción ha formado parte de un conjunto de actuaciones de diagnóstico, entre las que encontramos entrevistas a los principales agentes educativos del territorio y grupos de discusión con jóvenes, técnicos municipales, profesorado y un grupo mixto; sus resultados fueron presentados y trabajados a distintos niveles (familias, centros educativos y municipio). A pesar de la dificultad en la consecución de la participación de todos los agentes implicados, se han logrado resultados alentadores, ya que los distintos agentes han formulado propuestas a partir del análisis de la realidad del municipio y se han delimitado líneas estratégicas de intervención que se están llevando a la práctica.

Así, algunas de las propuestas concretas han sido: intensificar el trabajo conjunto para promover el conocimiento y la colaboración en diferentes ámbitos (formal, no formal, informal), incentivar la formación de los progenitores y profesionales que trabajan directamente con jóvenes, dinamizar la participación en acciones formativas y ofrecer la 
posibilidad a los participantes de ser partícipes en la planificación de las acciones, mejorar la circulación y sistematización de información sobre recursos e iniciativas existentes, promover una cultura de paz y proporcionar herramientas que ayuden a solucionar los conflictos de manera positiva a profesionales del ámbito educativo, social, de seguridad, a las familias y a los jóvenes. Debemos añadir que todas las acciones propuestas llevan consigo el valor de la participación como objetivo y estrategia para fomentar la convivencia positiva y la salud comunitaria.

A partir de este trabajo se ha iniciado un proceso de cambio que incide en las causas de la problemática abordada. Se observa el efecto que tienen las acciones promovidas y como estas retroalimentan a los participantes, lo que da lugar a nuevas dinámicas y relaciones. Además se han promovido y establecido nuevos esquemas de colaboración basados en el respeto mutuo y orientados hacia la búsqueda conjunta de soluciones para afrontar mejor problemáticas compartidas por familias, escuelas y comunidad. El DFVCC muestra la utilidad de la metodología dialéctica para implicar a la comunidad en el análisis de su realidad y aprovechar su energía para ayudar a transformarla y con ello, desde la construcción de una convivencia positiva y el trabajo cooperativo, alcanzar mayores cotas de bienestar, felicidad y favorecer el desarrollo óptimo de niños y niñas (Viguer, 2004).

Somos conscientes de las limitaciones del estudio, no solo por su carácter descriptivo y por los posibles sesgos de deseabilidad social de las respuestas, sino también porque los datos del mismo provienen de un contexto determinado, lo que puede suponer una dificultad a la hora de generalizar los resultados. Por otra parte, aún con sus limitaciones el DFVCC puede ser un instrumento de gran valor para que tanto las familias como los profesionales puedan analizar, tanto su manera de pensar como de actuar sobre algunos valores básicos. Y este análisis puede derivar en el diseño de acciones formativas dialógicas a nivel local implicando a los distintos agentes de la comunidad para avanzar en la consecución de modelos de familia y de convivencia óptimos. Son muchos los aspectos que requieren una atención más exhaustiva, pero este es un punto de partida sobre el que convendría seguir trabajando. En futuros trabajos abordaremos aspectos relativos a la socialización en la escuela y en la comunidad y se realizarán análisis multivariados que permitan valorar las relaciones entre las variables estudiadas.

\section{Referencias}

Ajuntament de Calella (2009). Parlem-ne. Debat familiar sobre coeducació. Educació en igualtat: què en diuen la infància i les famílies de Calella? Informe inédito.

Ajuntament de St. Feliu de Llobregat (2002). Informe de resultats sobre el debat familiar del projecte Sant Feliu. Disponible en http://www.santfeliu.org/ciutatencongres/final/deba.pdf

Alberich, T. (2000). Perspectivas de la investigación social. En T. Villasante, M. Montañes, \& J. Martí (Coords.). La investigación social participativa. Construyendo ciudadanía 1 (pp. 59-72). Barcelona: El Viejo Topo.

Álvarez González, M. R. (2006). Actitudes y opiniones de los españoles ante la infancia y la adolescencia. Madrid: Defensor del Menor en la Comunidad de Madrid.

Basagoiti, M. \& Bru, P. (2000). Mira quién habla. El trabajo con grupos en la IAP. En T. Villasante, M. Montañes, \& J. Martí (Coords.), La investigación social participativa. Construyendo ciudadanía 1(pp. 119-136). Barcelona: El Viejo Topo.

Chinman, M.; Hannah, G.; Wandersman, A.; Ebener, P.; Hunter, S.B.; Imm, P. \& Sheldon, J. (2005). Developing a community science research agenda for building community capacity for effective preventive interventions. American Journal of Community Psychology, 35(3-4), 143-157.

Comellas, M. J. (2006). Nuevas alternativas y modelos en la relación familia-escuela y profesionales de la comunidad. Cultura y Educación, 18, 295-311.

Consell Comarcal del Bages \& Universitat de València (2008). Informe tècnic de resultats sobre el Debat Familiar sobre Valors $i$ Convivència en els municipis del Bages Sud. Informe inédito.

Darling, N., \& Steinberg, L. (1993). Parenting style as context: an integrative model. Psychological Bulletin, 113, 487-498. 
Díaz-Aguado, M. J. (2003). Educación intercultural y aprendizaje cooperativo. Madrid: Pirámide.

Dryfoss, J., \& MacGuire, S. (2002). Inside Full Service Community School. California: Corwin Press.

Elzo, J., \& Orizo, F. A. (Eds.). (2000). España 2000, entre el localismo y la globalidad: La encuesta europea de valores en su tercera aplicación, 1981 - 1999. Madrid: S.M.

Flecha, J. R., \& Puigvert, L. (2002). Las comunidades de aprendizaje : Una apuesta por la igualdad educativa. REXE: Revista de estudios y experiencias en educación, 1(1), 11-20.

Fundació Jaume Bofill (2005). Síntesi de resultats del Debat Familiar: Què opinen les famílies sobre les activitats educatives fora de l'horari escolar? Barcelona: Ajuntament de Barcelona. Disponible en: http://www.laboratorideltemps.org/media/0000001000/0000001048.pdf

González Blasco, P. (Dir.)., González- Anleo, J.; Elzo, J.; González-Anlo Sánchez, J.M.; López Ruiz, J.A., \& Valls, M. (2006). Jóvenes españoles 2005. Madrid: S.M.

Hernández, M.; Gómez, I.; Martín, M. J., \& González, C. (2008). Prevención de la violencia infantil juvenil: estilos educativos de las familias como factores de protección. International Journal of Psychology and Psychological Therapy, 8, 73-84

Jares, X. R. (2006). Pedagogía de la convivencia. Barcelona: Graó.

Meil, G. (2006). Padres e hijos en la España actual. Barcelona: Fundación "La Caixa".

Musitu, G., \& García, F. (2004). Las consecuencias de la socialización en la cultura española. Psicothema, 16, 288-293.

Musitu, G., Estévez, E., Jiménez, T., \& Herrero, J. (2007). Familia y conducta delictiva y violenta en la adolescencia. En S. Yubero, E. Larrañaga, \& A. Blanco (Coords.). Convivir con la violencia (pp. 135-150). Cuenca: Universidad de Castilla la Mancha.

Musitu, G., Jiménez, T., \& Povedano, A. (2009). Familia y escuela: escenarios de riesgo y protección en la violencia escolar. REME, 12, 32-33.

Navas, L., Torregrosa, G., \& Mula, A. (2004). Algunas variables predictoras de las actitudes del alumnado ante la integración escolar. Revista de Psicología Social, 19 (2), 159-171.

Ortega, P., \& Mínguez, R. (2001). Familia y transmisión de valores.Murcia: Universidad de Murcia. Disponible en: http://www.mercaba.org/ARTICULOS/F/ familia_y_transmision_de_valores.htm

Pichardo, M.C. (2000). Influencia de los estilos educativos de los padres y del clima social familiar en la adolescencia temprana y media. Granada: Servicio de Publicaciones de la Universidad de Granada.

Pichardo, M. C., Justicia, F., \& Fernández, M. (2009). Prácticas de crianza y competencia social en niños de 3 a 5 años. Pensamiento Psicológico, 13(6), 37 - 47.

Ribes, C. (2002). Dos contextos educativos: familias y profesionales. Aula de Innovación Educativa, 108, 24-28.

Rodrigo, M. J., \& Palacios, J. (Coords.) (1998). Familia y desarrollo humano. Madrid: Alianza.

Viguer, P. (2004). Optimización Evolutiva. Fundamentos del desarrollo óptimo. Madrid: Pirámide.

Viguer, P., \& Avià, S. (2009). Un modelo local para la promoción de la convivencia y la prevención de la violencia entre iguales desde el ámbito comunitario. Cultura y Educación, 21(3), 345-349.

Viguer, P., Avià, S. \& Rossell, A. (2013). Labordatge integral de les violències que afecten les persones joves: un model d'intervenció local de promoció de la convivència i prevenció de les violències. Barcelona: Generalitat de Catalunya, Departament de Benestar Social i Família, Direcció General de Joventut.

Viguer, P., \& Solé, N. (2011). Debate familiar sobre valores y convivencia: una investigación participativa para implicar a las familias en el análisis y la transformación de su realidad. Cultura y Educación, 23(1), 105-118.

Viguer, P., \& Solé, N. (2012). Escuela e iguales como contextos de socialización en valores y convivencia: una investigación participativa a través de un debate familiar. Cultura y Educación, 24(4), 475-487.

Viguer, P., Rodrigo, M. F., \& Solé, N. (2013). The family debate on values and living together: a community-based approach through participatory action research. Journal of Community Psychology, 41, 944-958. Doi:10.1002/jcop.21582. 